\title{
Carcinoma familiar de tiroides no medular. Reporte de un caso clínico y revisión de la literatura
}

\author{
Familial non medullary thyroid carcinoma. Report of a case and literature review
}

\author{
Valentina Bustos $\mathbf{G}^{1}$, Jaime Bermeo $\mathrm{S}^{2}$, Cristian Papuzinski $\mathrm{A}^{2}$, Daniel Moyano $\mathbf{M}^{2}$.
}

\begin{abstract}
RESUMEN
El carcinoma tiroideo familiar no medular (CFTNM) representa aproximadamente entre el 3,2\% y 9,6\% de todos los cánceres de tiroides, y se define por la presencia de cáncer diferenciado de tiroides en 2 o más familiares, en ausencia de otros síndromes familiares conocidos o exposición a radiación. Si bien su fisiopatología es aún incierta, algunos investigadores postulan un patrón de herencia dominante con penetrancia incompleta, no habiendo aún un gen específico responsable. Esta entidad suele presentarse a una menor edad y con características más agresivas que en su forma esporádica. Dado el interés por conocer la presentación de esta enfermedad y las recomendaciones para su manejo, se presenta el caso de una paciente diagnosticada con cáncer papilar de tiroides con el antecedente de 4 familiares con la misma patología. Actualmente el tamizaje mediante ecografía cervical y biopsia por punción aspiración con aguja fina de los nódulos tiroideos es el examen de elección ante la presencia del antecedente de CFTNM, ya que aún no hay estudios genéticos disponibles. La tiroidectomía total más disección ganglionar es el tratamiento de elección. Debido al comportamiento más agresivo y peor pronóstico del CFTNM, es necesaria un alto índice de sospecha y una investigación completa en la presencia de un componente familiar.

Palabras clave: Cáncer tiroideo familiar, carcinoma familiar no medular de tiroides, cáncer de tiroides.
\end{abstract}

\begin{abstract}
The non-familial medullary thyroid carcinoma (FNMTC) represents approximately between 3.2 and $9.6 \%$ of all thyroid cancers, and is defined by the presence of differentiated thyroid cancer in 2 or more families in the absence of other known familial syndromes or radiation exposure. Although the pathophysiology is still uncertain, some investigators posit a dominant pattern of inheritance with incomplete penetrance, but
\end{abstract}

\footnotetext{
1 Interna de Medicina, Universidad de Valparaíso.

2 Médico Otorrinolaringología, Hospital Carlos Van Buren, Universidad de Valparaíso.
} 
still there is no specific gene responsible. It occurs at a younger age and with more aggressive characteristics than the sporadic form. Because of the interest in learning about the presentation of this disease and its recommendations, we present the case of a patient diagnosed with papillary thyroid cancer with a history of 4 family with the same pathology. Actually cervical screening by ultrasound and the fine needle aspiration biopsy (FNAB) of thyroid nodules is the examination of choice in the presence of a history of FNMTC, since no genetic studies yet available. Total thyroidectomy with lymph node dissection is the treatment of choice. Because the more aggressive behavior and poor prognosis of FNMTC, a high index of suspicion and a full investigation is required in the presence of a familial component.

Key words: Family thyroid cancer, familial non medullary thyroid carcinoma, thyroid cancer.

\section{INTRODUCCIÓN}

El cáncer de tiroides corresponde a la neoplasia endocrina más frecuente ${ }^{1}$. Su aparición se ha acrecentado a más del doble en los últimos 30 años ${ }^{1}$, estimándose una incidencia en Chile mayor a 7,86 casos por 100.000 habitantes en el año $2011^{2}$. Esta neoplasia, se puede clasificar según las células que lo originan, siendo medulares, los producidos por células parafoliculares encargadas de la producción de calcitonina, y no medulares, los originados en las células foliculares de la tiroides, dentro de los cuales tenemos papilares, foliculares y anaplásicos; estos últimos constituyen el $90 \%$ de los cánceres de esta glándula 3 . Ambos tipos, medulares y no medulares, pueden tener una presentación esporádica o familiar ${ }^{4,5}$. La forma familiar del carcinoma medular, corresponde al $25 \%$ de los casos y es por lo general, un componente de neoplasias endocrinas múltiples tipo 2 (NEM2), 0 se puede presentar como patología única, estando las gatillantes genéticas de estos tumores bien establecidas $^{6}$. Por otro lado, el carcinoma no medular si bien se presenta mayoritariamente de forma aislada, se ha descrito que puede presentarse como una enfermedad de origen familiar, pudiendo asociarse a síndromes hereditarios conocidos, tales como: poliposis adenomatosa familiar, síndrome de Cowden, complejo de Carney tipo 1, síndrome de Werner, síndrome de Gardner, síndrome de Peutz-Jeghers y NEM 2.

El carcinoma tiroideo familiar no medular (CFTNM), no asociado a entidades sindromáticas, corresponde a una patología poco definida hasta la fecha ${ }^{3,6}$. Ésta se define clínicamente como la presencia de cáncer de tiroides diferenciado en dos 0 más familiares de primer o segundo grado, excluyendo los casos que forman parte de algún síndrome genético familiar o que hayan sido expuestos a factores ambientales predisponentes ${ }^{7,8}$. Esta entidad fue descrita por primera vez en 1955 por Robinson y Orr en gemelos monocigóticos ${ }^{9}$, y se estima que correspondería al 3,2\%-9,6\% de los cánceres de tiroides, aunque su prevalencia exacta es aún incierta ${ }^{3,6}$. Su fisiopatología es aún indeterminada, por lo que el diagnóstico es exclusivamente mediante la historia y antecedentes familiares, ya que no se cuenta con características clínicas o exámenes que nos permitan diferenciarlo de una presentación esporádica ${ }^{3}$. Algunos investigadores postulan un patrón de herencia dominante con penetrancia incompleta, no habiendo aún un gen determinado como responsable, pese a que existen varias regiones génicas candidatas como implicadas, entre las que destacan: MNG1 (14q32), 19p13.2, 1q21, 2q21, 8p23.1-p22 y el complejo telomerasa-telomerasa ${ }^{3,7,8}$. Sin embargo, tampoco se descarta una etiología poligénica ${ }^{3}$. La mayoría de los CFTNM son papilares, y al igual que en las presentaciones esporádicas, se ha visto que es 2 a 3 veces más frecuente en el sexo femenino. El riesgo de tener cáncer tiroideo puede aumentar 3,2 a 6,4 veces al haber antecedente familiar, dependiendo del sexo y el grado de parentesco, siendo mayor el riesgo para las hermanas ${ }^{6,9}$.

Con respecto a la presentación clínica de CFTNM, se ha descrito que la edad media de inicio es menor que en la presentación esporádica (39 
a 43 años versus 46 a 49 años), no quedando claro si corresponde a una característica biológica propia o es consecuencia del sesgo que genera una pesquisa más acuciosa al haber antecedentes familiares de cáncer6,10,11. También se debe considerar el fenómeno de anticipación clínica, donde las segundas generaciones afectadas tendrían una presentación a edad más temprana y de características más agresivas, como lo son la invasión extratiroidea y una mayor tasa de recurrencia ${ }^{12}$. Algunos investigadores han reportado tasas de tumores multicéntricos más altas, mayor número de metástasis a ganglios linfáticos, invasión vascular, mayor invasión local y tasas de reintervención y recurrencia más altas, en comparación a las presentaciones esporádicas ${ }^{4,9}$. Sin embargo, esto es aún controvertido, debido a que múltiples estudios no

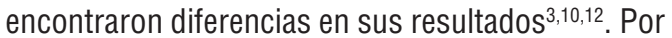
otra parte, los familiares de pacientes con CFTNM corren mayor riesgo de desarrollar enfermedades benignas de la tiroides: bocio multinodular (BMN), tiroiditis de Hashimoto, adenomas foliculares ${ }^{5}$.

Actualmente en Chile, existen pocos datos acerca de la presentación de CFTNM, siendo todavía una entidad rara de ver en los centros encargados de manejar el cáncer de tiroides ${ }^{6}$. No hay guías clínicas en nuestro país que determinen el adecuado proceder ante estos casos, por lo que la decisión es tomada según el criterio del equipo médico de cada centro.

Dado el interés por conocer sobre la presentación de esta entidad y la importancia de la pesquisa de los antecedentes familiares en el cáncer de tiroides, se decide presentar el caso de una paciente atendida el año 2016 en el Servicio de Otorrinolaringología del Hospital Carlos Van Buren, diagnosticada con cáncer papilar de tiroides.

\section{CASO CLÍNICO}

Paciente de sexo femenino de 51 años de edad, con antecedente familiar de carcinoma papilar de tiroides en dos hermanas y dos sobrinos (Figura 1). Consulta en extrasistema por cuadro de aumento de volumen en región cervical anterior, y al examen físico se palpa un bocio multinodular, sin adenopatías a la palpación. Se solicita estudio de hormonas tiroideas, incluyendo hormona tiroestimulante (TSH) ultrasensible, T4 libre y T3, ecografía cervical, y radiografía de tórax. La ecografía cervical mostró un aumento de volumen tiroideo a expensas del lóbulo derecho de 1,9 por 2,2 cms, con una lesión isoecogénica mal definida con componentes quísticos en su interior, y aumento de vascularización al estudio doppler; además lesiones de aspecto adenopático a nivel supraclavicular bilateral y supraesternal, siendo la mayor 2,6 por 1,7 cms. La función tiroidea resultó conservada y el resto de exámenes sin alteraciones. Se realiza a la semana siguiente punción con aguja fina (PAAF) en una de las adenopatías, que resultó compatible con una metástasis ganglionar de un carcinoma papilar de tiroides. Es derivada al Hospital Carlos Van Buren para evaluación del caso y decidir el manejo. Se decide la realización de tiroidectomía total más disección cervical ganglionar selectiva de grupos IV derecho e izquierdo y grupo VI, la cual se realizó sin

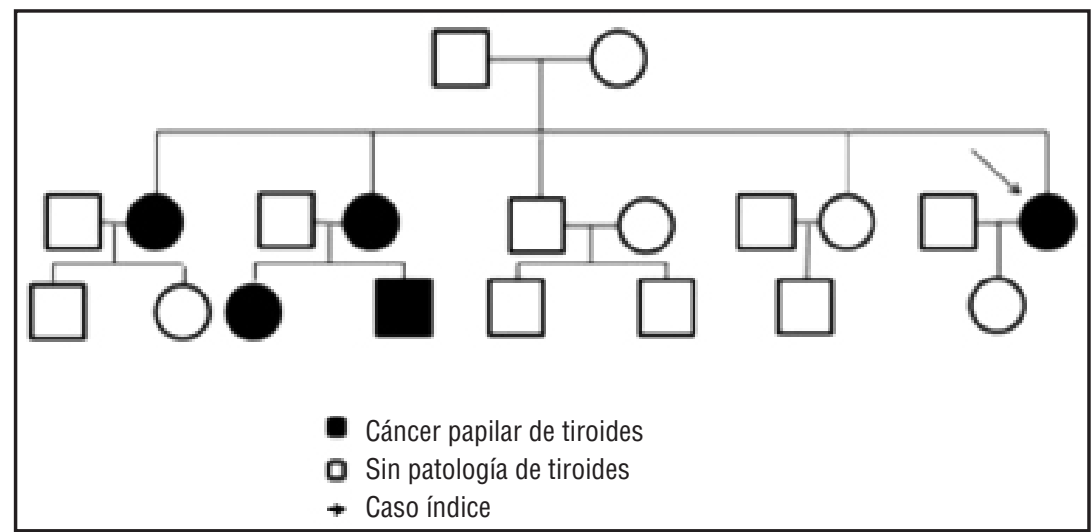

Figura 1. Genograma del caso. 
complicaciones intraoperatorias. La biopsia reveló un carcinoma papilar de tiroides y metástasis en 4 de 4 ganglios, sin invasión extracapsular. La paciente evoluciona favorablemente, normocalcémica, sin apremio ventilatorio, sin disfagia y con leve disfonía. Es dada de alta y presentada en Comité de Endocrinología, donde se decide terapia con radioyodo $131 \mathrm{de} 200 \mathrm{mci}$. Actualmente en buenas condiciones generales, en espera de tratamiento con yodo radioactivo.

\section{DISCUSIÓN}

Se describe un caso de 5 miembros afectados por cáncer de tiroides en la misma familia, en tan sólo dos generaciones. La descripción de la literatura señala que se ha estimado que entre el $31 \%$ y $38 \%$ de los miembros de las familias con dos miembros afectados son propensos a portar el rasgo familiar, siendo el resto esporádico, sin embargo, en las familias con tres o más miembros afectados con CFTNM, la probabilidad de poseer el rasgo familiar es del $96 \%$, por lo que autores plantean que los múltiples resultados controvertidos en relación a la presentación y agresividad de la CFTNM se debe a la sobreestimación de esta entidad, incluyendo dentro de la definición muchos casos que pueden corresponder a presentaciones esporádicas ${ }^{3,13}$. Además, estudios han demostrado que al haber más de dos miembros afectados en la familia, la supervivencia libre de enfermedad es significativamente menor, siendo más agresiva que otras presentaciones familiares, justificándose esfuerzos por un diagnóstico precoz y el tratamiento oportuno en estas familias $9,11$.

Actualmente, en relación al CFTNM, cualquier recomendación es categoría $C$, debido a que la mayoría de la evidencia se basa en estudios retrospectivos, casos y controles y a opiniones de expertos $^{5}$. Se debe considerar en el manejo el número de miembros afectados y la naturaleza de la enfermedad dentro de la familia. Si bien no hay estudios de costo efectividad realizados, se sugiere que cualquier familia con 20 más miembros con cáncer no medular de tiroides no sindromático debe ser sometida a un tamizaje con ecografía cervical, incluso si son asintomáticos ${ }^{3,5}$. Se debe centrar la historia en los antecedentes familiares, exposición a radiación o a dieta deficitaria en yodo y patología tiroidea, tanto benigna como maligna. Es importante indagar en la presencia de síntomas compresivos como disnea, disfonía o disfagia y la presencia de otras patologías que puedan configurar un síndrome, como por ejemplo poliposis adenomatosa familiar, Cowden, complejo de Carney tipo 10 Werner $^{3}$. Se debe considerar una ecografía cervical en todos los miembros de la familia y en caso de encontrarse nódulos tiroideos, el estudio mediante punción con aguja fina guiada por ecografía es el examen de elección. Sin embargo, se ha mostrado una mayor tasa de falsos positivos en estos pacientes debido a la también mayor incidencia de patología benigna en este grupo de pacientes $^{3}$. La edad de inicio del tamizaje no está clara, sin embargo se sugiere a los 20 años en asintomáticos 010 años antes de la edad de pesquisa del familiar que haya sido diagnosticado más joven. Inclusive, se recomienda realizar el tamizaje con el antecedente de un solo familiar o muchas presentaciones benignas dentro de una misma familia ${ }^{3}$. Como lamentablemente aún ningún gen ha sido determinado como el responsable de CFTNM, no hay pruebas genéticas disponibles ${ }^{14-16}$.

Con respecto al tratamiento, la tiroidectomía total es la elección en el paciente diagnosticado de CFTNM, considerándose la disección ganglionar anterior de rutina en tumores $>1 \mathrm{~cm}$, sospecha ecográfica de compromiso ganglionar, comportamiento agresivo de la enfermedad en otros miembros de la familia o presencia de 30 más miembros de la familia afectados ${ }^{3}$. El uso de radioyodo 131 sería recomendable en todos los casos, sobre todo en los casos de comportamiento más agresivo de la enfermedad o más de 3 miembros afectados. La tiroidectomía profiláctica está aún en discusión, sin embargo se justificaría en pacientes con nódulo tiroideo y dos o más familiares afectados, incluso en tamaños menores a $1 \mathrm{~cm}^{3}$.

\section{CONCLUSIÓN}

Debido al comportamiento más agresivo y peor pronóstico del CFTNM en relación a la presentación esporádica, es necesaria una indagación profunda por parte del equipo médico en la presencia de un componente familiar que pudiera estar presente en 
Ios pacientes. Casos como el presentado muestran la importancia de contar con un tamizaje que permita pesquisar tempranamente la presencia o mayor riesgo de cáncer de tiroides en los miembros de familias con antecedentes familiares. Lo anterior permitiría iniciar tempranamente el estudio diagnóstico, realizar un correcto estadio y definir un manejo óptimo. Es preciso, por lo mismo, nuevas investigaciones, idealmente prospectivas que nos permitan comprender la presentación de

\section{BIBLIOGRAFÍA}

1. Cramer JD, PFu, Harth K, Margevicius S, Wilhelm SM. Analysis of the rising incidence of thyroid cancer using the Surveillance, Epidemiology and End Results national cancer data registry. Surgery 2010; 148: 1147-53.

2. Sapunar J, Muñoz S \& Roa JC (2014). Epidemiología del cáncer de tiroides en Chile: Resultados del estudio INCATIR. Revista Médica de Chile 142(9): 1099-105.

3. Mazeh H, Sippel R. Familial Nonmedullary Thyroid carcinoma. Thyroid 2013; 23 (9): 1049-56.

4. Nosé V. Familial Non-Medullary Thyroid Carcinoma: An Update. Endocr Pathol 2008; 19: 226-40.

5. Nosé V. (2011). Familial thyroid cancer: a review. Modern Pathology 24, S19-S33.

6. Sippel R, Caron N, Clark 0. An Evidence-based Approach to Familial Nonmedullary Thyroid Cancer: Screening, Clinical Management, and Follow-uP. World J Surg 2007; 31: 924-33.

7. Mosso L, Velasco S, Salazar I, Solar A, González H, Cardona B, ... \& Cruz, F. (2007). Carcinoma familiar del tiroides no medular (CFTNM): características de presentación en 17 casos. Revista Médica de Chile 135(6): 718-24.

8. Malchoff CD \& Malchoff DM (2006). Familial nonmedullary thyroid carcinoma. Cancer Control 13(2), 106.

9. Ferrada C, Godoy C, Martínez A, García H (2014). Cáncer tiroideo papilar: Reporte de 4 casos esta entidad, su comportamiento, y conocer en un futuro los genes específicos implicados en la fisiopatología de la enfermedad. Futuros estudios en nuestro país podrían enfocarse en diferenciar el comportamiento del cáncer familiar no medular de tiroides, según el número de miembros afectados, lo que colaboraría en determinar los casos que efectivamente posean un componente hereditario, diferenciándolos de presentaciones esporádicas dentro de un mismo grupo familiar.

familiares. Revista Chilena de Pediatría 85(3), 351-8.

10. Robinson D, OrR T. Carcinoma of the thyroid and other diseases of the thyroid in identical twins. Arch Surg 1955; 70: 923-8.

11. Hemminki K, Eng C \& Chen B (2005). Familial risks for nonmedullary thyroid cancer. The Journal of Clinical Endocrinology \& Metabolism 90(10): 5747-53.

12. Moses W, Weng J \& Kebebew E. (2011). Prevalence, clinicopathologic features, and somatic genetic mutation profile in familial versus sporadic nonmedullary thyroid cancer. Thyroid 21(4): 367-71.

13. Park YJ, Ahn HY, Chol HS, Kim KW, Park DJ \& Сно BY. (2012). The long-term outcomes of the second generation of familial nonmedullary thyroid carcinoma are more aggressive than sporadic cases. Thyroid 22(4): 356-62.

14. Pitola F, Cross G, Salvai ME, Abelleira E \& Niepomniszcze $H$ (2011). Patients with familial non- medullary thyroid cancer have an outcome similar to that of patients with sporadic papillary thyroid tumors. Arquivos Brasileiros de Endocrinologia \& Metabologia 55(3): 219-23.

15. Fan YF, Zhang B, Yang X, Shang ZH, Liu HF, Xie Y., ... \& LI XY (2015). Clinicopathologic Features of Familial Nonmedullary Thyroid Carcinoma. Chinese Medical Journal 128(8): 1037.

16. Khan A, Smellie J, Nutting C, Harrington K, Newbold K. Familial Nonmedullary Thyroid Cancer a Review of the Genetics. Thyroid 2010; 20 (7): 795-801.

Dirección: Jaime Bermeo S.

Hospital Carlos Van Buren, Valparaíso, Chile

E mail: Jaime@bermeos.net 